Proceedings of the Institute of Mathematics and Mechanics,

National Academy of Sciences of Azerbaijan

Volume 47, Number 2, 2021, Pages 215-225

https://doi.org/10.30546/2409-4994.47.2.215

\title{
SCALABILITY OF G-FRAMES BY DIAGONAL OPERATORS
}

\author{
AHMAD AHMADI AND ASGHAR RAHIMI
}

\begin{abstract}
Tight frames are similar to orthogonal bases, except that the frame coefficients are not unique, but they are stable in calculations and numerical algorithms. Not all frames are tight frames, but some have the ability to become tight frames. These frames are called scalable frames. In this article, we extend this good property of frames to $G$-frames. For this purpose, we define the scalable $G$-frame based on the diagonal operators, and obtain a preconditioner for its analysis operator by block diagonal operator. We also provide the necessary and sufficient conditions for the scalability of the $G$-frames based on the frames induced by the $G$-frames.
\end{abstract}

\section{Introduction}

The frames are a generalization of orthogonal bases, with the difference that they are not linearly independent. That is, unlike the bases, the coefficients of the frames are not necessarily unique. Gabor [11] formulated a new method for signal decomposition and signal expansion based on preliminary signals. According to this study, Duffin and Schaeffer [8] provided frames for the Hilbert space to solve the non-harmonic series. Until 1980, the importance of frames was not known. After Daubechies, Grossmann, and Meyer [7] reintroduced the frames, a lot of research was done on this subject. Various extensions and generalizations of frames have been introduced so far, such as pseudo-frames [15] quasi projectors [10] and oblique frames [5]. These frames are a special feature of $G$-frames, which was first introduced by Sun [17].

The applications of frames can be divided into two categories. In the first applications, the frame is used for data analysis. In this case, the goal is to resist data deletion, data analysis, and compression. Other applications are data extensions. The approach is used in summarizing methods such as compressing sensors. In applications, there need to be stable numerical algorithms. Tight frames are one of the best subclasses of frames in this situation. One may ask how a tight frame can be obtained. One of the solutions that can be used to get a tight frame from a frame is the effect of the operator $S_{F}^{-\frac{1}{2}}$ on vectors of the frame $F=\left\{f_{k}\right\}$, where $S_{F}$ is frame operator for $F$. But it's not easy to get this operator, and also make changes to the frame vectors. One of the most important

2010 Mathematics Subject Classification. 42C15, $15 \mathrm{~A} 69$.

Key words and phrases. Scalable frame, $G$-frame, Diagonal operator, Condition number. 
ways to get a tight frame is to change the scale of each frame vector. For this purpose, Kutyniok and et al. [13] introduced a type of frames, which by scaling of its vectors, become Parseval frames. They called these type of frames scalable frames. They showed that for transforming a frame $F=\left\{f_{k}\right\}$ into a scalable frame, one should find a diagonal operator $D$ such that the conditional number of the operator $D T_{F}^{*}$, where $T_{F}^{*}$ is analysis operator of $F$, is one. In addition, they stated, with a geometric interpretation, that not all frames are necessarily scalable. After that, scalable frames have been investigated by several researchers $[3,13,14]$.

In this study, we develop the concept of the scalability of frames to $G$-frames, and state the scalability of $G$-frames using non-negative diagonal operators instead of non-negative numbers for scale changes. Furthermore, we use block diagonal operator to precondition $G$-frames.

The present paper is organized as follows: In Section 2, we fix the notations of this paper and summarize some results needed for the rest of the paper. In Section 3, we present the concept of scalability of $G$-frames and prove some of the proper results for these $G$-frames. Finally, in the last section, we introduce some explicit constructions of scalable $G$-frames.

\section{Notation and Preliminaries}

Throughout this paper $\mathcal{K}$ and $\mathcal{H}$ are two Hilbert spaces and $\left\{\mathcal{H}_{i}, i \in I\right\}$ and $\left\{\mathcal{K}_{i}, i \in I\right\}$ are sequences of Hilbert spaces, where $I \subset \mathbb{Z} . \mathcal{L}(\mathcal{K}, \mathcal{H})$ is the collection of all bounded linear operators from $\mathcal{K}$ to $\mathcal{H}$ and for brevity, we denote $\mathcal{L}(\mathcal{H}, \mathcal{H})$ by $\mathcal{L}(\mathcal{H})$. We also recall that the space

$$
\left(\bigoplus \mathcal{H}_{i}\right)_{l^{2}}=\left\{\left\{x_{i}\right\} \mid x_{i} \in \mathcal{H}_{i}, i \in I: \quad \sum_{i \in I}\left\|x_{i}\right\|_{2}^{2}<\infty\right\},
$$

is a Hilbert space with pointwise operations and the inner product as

$$
\left\langle\left\{x_{i}\right\},\left\{y_{i}\right\}\right\rangle=\sum_{i \in I}\left\langle x_{i}, y_{i}\right\rangle
$$

Definition 2.1. An operator $D$ defined on the (closed) linear span of a basis $\left\{e_{i}\right\}_{i \in I}$ in a normed space $X$ is called diagonal operator whenever $D e_{k}=\lambda_{k} e_{k}$, where $k \geq 1$ and $\lambda_{k}$ 's are complex numbers. If $D$ is a continuous operator, one has

$$
\begin{gathered}
\sup _{k \geq 1}\left|\lambda_{k}\right|<\infty . \\
\|D\|=\sup _{k \geq 1}\left|\lambda_{k}\right|<\infty .
\end{gathered}
$$

We denote the domain, the kernel, and the range of linear operator $S$ by $\operatorname{dom} S$, ker $S$ and $\operatorname{rang} S$, respectively.

Definition 2.2. Assume that $D_{i}$ is an operator on $\mathcal{H}_{i} ; i \in I$. We say that the operator $D$ on $\left(\bigoplus \mathcal{H}_{i}\right)_{l^{2}}$ is a block diagonal operator with $\left\{D_{i}\right\}$ as its diagonal, whenever

$$
D\left(\left\{f_{i}\right\}\right)=\left\{D_{i} f_{i}\right\}, \quad\left\{f_{i}\right\} \in \operatorname{dom} D,
$$


where,

$$
\operatorname{dom} D=\left\{\left\{f_{i}\right\} \in\left(\bigoplus \mathcal{H}_{i}\right)_{l^{2}}:\left\{D_{i} f_{i}\right\} \in\left(\bigoplus \mathcal{H}_{i}\right)_{l^{2}}\right\}
$$

For the block diagonal operator $D$, we recall that $\|D\|=\sup _{i \in I}\left\|D_{i}\right\|$.

Definition 2.3. An operator $S: \mathcal{H} \rightarrow \mathcal{K}$ will be called ICR (Injective Closed Range), whenever $S$ has the following properties

i) $S$ is closed linear operator,

ii) there exists $r>0$ such that $\|S f\| \geq r\|f\|, \quad \forall f \in \mathcal{H}$.

An operator $P$ on the Hilbert space $\mathcal{H}$ is called non-negative operator if $\langle P f, f\rangle \geq 0$. If $P$ is non-negative, then its eigenvalues are non-negative real numbers [6].

2.1. Scalable frames. Here's a brief overview of topics on frames and scalable frames. For more information on frames see $[2,4]$ and for scalable frames see $[1,13,14,16,18]$.

Definition 2.4. A sequence $\left\{f_{n}\right\}$ in a Hilbert space $\mathcal{H}$ is called a frame if there exist two constants $0<A \leq B<\infty$ such that

$$
A\|f\|^{2} \leq \sum_{n \in \mathbb{N}}\left|\left\langle f, f_{n}\right\rangle\right|^{2} \leq B\|f\|^{2} \quad \forall f \in \mathcal{H} .
$$

If $A=B$ the sequence $\left\{f_{n}\right\}$ is called tight frame and if $A=B=1$, it is called Parseval frame.

Let $F=\left\{f_{n}\right\}$ be a frame, the map

$$
T_{F}: l_{2}(\mathbb{N}) \rightarrow \mathcal{H} ; \quad T_{F}\left(\left\{c_{n}\right\}\right)=\sum_{n \in \mathbb{N}} c_{n} f_{n},
$$

is called the synthesis operator of the frame $F$. This operator is bounded and onto. The adjoint $T_{F}$ denoted by

$$
T_{F}^{*}: \mathcal{H} \rightarrow l_{2}(\mathbb{N}) ; \quad T_{F}^{*}(f)=\left\{\left\langle f, f_{n}\right\rangle\right\},
$$

is called analysis operator of $F$. The operator

$$
S_{F}: \mathcal{H} \rightarrow \mathcal{H} ; \quad S_{F} f=T_{F} T_{F}^{*} f=\sum_{n \in \mathbb{N}}\left\langle f, f_{n}\right\rangle f_{n},
$$

is called frame operator. For the frame $F$, the frame operator is bounded, selfadjoint, positive and invertible.

Definition 2.5. A frame $F=\left\{f_{i}\right\}_{i \in I}$ is called scalable frame whenever there exists a sequence of non-negative real numbers $\left\{\alpha_{i}\right\}_{i \in I}$ such that $\left\{\alpha_{i} f_{i}\right\}_{i \in I}$ is a Parseval frame. Also, if $\alpha_{i}$ 's are positive numbers then the frame $F=\left\{f_{i}\right\}_{i \in I}$ is called strictly scalable.

Let $F=\left\{f_{i}\right\}_{i \in I}$ be a frame for Hilbert space $\mathcal{H}$. Then $F$ is scalable if and only if there exists a non-negative diagonal operator $D$ on $l^{2}(\mathbb{I})$ such that $\overline{T_{F}^{*} D} D T_{F}=I_{\mathcal{H}}$, [13]. 
Theorem 2.1. [13] Let $F=\left\{f_{i}\right\}_{i=1}^{M} \subset \mathbb{R}^{N} \backslash\{0\}$ be a frame for $\mathbb{R}^{N}$. Then $F$ is not scalable if and only if there exists a symmetric matrix $Y \in \mathbb{R}^{N \times N}$ with $\operatorname{tr}(Y)<0$ such that $f_{j}^{T} Y f_{j} \geq 0$ for all $j=1, \ldots, M$, where $f_{j}^{T}$ denotes the transpose of the matrix $f_{j}$.

2.2. $G$-frames. In this section, we present the basic concepts of the $G$-frames. As we mentioned earlier, this notation has been introduced by Sun [17]. For more details see $[5,12,17]$.

A sequence $\left\{G_{i} \in \mathcal{L}\left(\mathcal{H}, \mathcal{H}_{i}\right)\right\}_{i \in I}$ is called $G$-frame for $\mathcal{H}$ with respect to $\left\{\mathcal{H}_{i}\right\}_{i \in I}$ whenever there exist two constants $A, B$ such that $0<A \leq B<\infty$ and

$$
A\|f\|^{2} \leq \sum_{i \in I}\left\|G_{i} f\right\|^{2} \leq B\|f\|^{2}, \quad \forall f \in \mathcal{H} .
$$

The sequence $\left\{G_{i}\right\}_{i \in I}$ is called tight $G$-frame whenever $A=B$, if $A=B=1$, then $\left\{G_{i}\right\}_{i \in I}$ is called Parseval $G$-frame. Also, if the right hand (2.1) holds then the sequence $\left\{G_{i}\right\}_{i \in I}$ is called G-Bessel.

We recall that the synthesis operator for $G$-frame $\mathcal{G}=\left\{G_{i}\right\}_{i \in I}$ is

$$
T_{\mathcal{G}}:\left(\bigoplus \mathcal{H}_{i}\right)_{l^{2}} \rightarrow \mathcal{H} ; \quad T_{\mathcal{G}}\left(\left\{f_{i}\right\}_{i \in I}\right)=\sum_{i \in I} G_{i}^{*} f_{i}
$$

and the analysis operator is

$$
T_{\mathcal{G}}^{*}: \mathcal{H} \rightarrow\left(\bigoplus \mathcal{H}_{i}\right)_{l^{2}} ; \quad T_{\mathcal{G}}^{*} f=\left\{G_{i} f\right\}_{i \in I} .
$$

Now we are ready to recall the definition of $G$-frame operator

$$
S_{\mathcal{G}}: \mathcal{H} \rightarrow \mathcal{H} ; \quad S_{\mathcal{G}} f=\sum_{i \in I} G_{i}^{*} G_{i} f .
$$

A $G$-frame operator is bounded, self-adjoint and invertible operator and the family $\left\{G_{i} S^{-1}\right\}_{i \in I}$ is called the canonical G-dual frame.

Suppose that $\left\{G_{j}\right\}_{j \in I}$ is a $G$-frame and $\left\{e_{j, k}: k \in \mathbb{K}_{j}\right\}$, where $\mathbb{K}_{i} \subseteq \mathbb{Z}$, is an orthonormal basis for $\mathcal{H}_{j}$. The sequence $\left\{u_{j, k}=G_{j}^{*} e_{j, k}: j \in I, k \in \mathbb{K}_{j}\right\}$ is called the frame induced by $\left\{G_{j}\right\}_{j \in I}$ with respect to $\left\{e_{j, k}: k \in \mathbb{K}_{j}\right\}$ [Theorem 3.1 [17]].

\section{Scalibility of $G$-frames}

In this section, we provide a new definition for the scalability of $G$-frames. For this definition, we use diagonal operators and block diagonal operators.

At first the question arises: if any sequence of operators which affects a $G$-frame creates a $G$-frame again. The answer to this question is certainly no. Below, we describe the conditions under which after the effect of a sequence of operators on a $G$-frame, a new $G$-frame is created.

A positive diagonal operator $D \in \mathcal{L}(\mathcal{H})$ is invertible, thus there exist two constants $K, K^{\prime}$ such that

$$
K\|f\| \leq\|D f\| \leq K^{\prime}\|f\| \quad \forall f \in \mathcal{H} .
$$

Definition 3.1. A sequence of positive diagonal operators $\left\{D_{i} \in L\left(\mathcal{H}_{i}\right)\right\}_{i \in I}$ is called semi-normalized whenever

$$
0<i n f_{i \in I} K_{i} \leq \sup _{i \in I} K_{i}^{\prime}<\infty,
$$


where $K_{i}$ and $K_{i}^{\prime}$ are the lower bound and the upper bound for $D_{i}, i \in I$, respectively.

Lemma 3.1. Let $\left\{G_{i} \in \mathcal{L}\left(\mathcal{H}, \mathcal{H}_{i}\right)\right\}_{i \in I}$ be a $G$-frame for the Hilbert space $\mathcal{H}$ with respect to $\left\{\mathcal{H}_{i}\right\}_{i \in I}$ with bounds $A, B$ and $\left\{D_{i} \in L\left(\mathcal{H}_{i}\right)\right\}_{i \in I}$ be a sequence of seminormalized diagonal operators. Then $\left\{D_{i} G_{i}\right\}_{i \in I}$ is a $G$-frame.

Proof. Let $f \in \mathcal{H}$. Then

$$
\begin{gathered}
\sum_{i \in I}\left\|D_{i} G_{i} f\right\|^{2} \leq \sum_{i \in I}\left\|D_{i}\right\|^{2}\left\|G_{i} f\right\|^{2} \leq \\
\sup _{i \in I} K_{i}^{\prime} \sum_{i \in I}\left\|G_{i} f\right\|^{2} \leq \operatorname{Bsup}_{i \in I} K_{i}^{\prime}\|f\|^{2} .
\end{gathered}
$$

On the other hand,

$$
\begin{gathered}
\sum_{i \in I}\left\|D_{i} G_{i} f\right\|^{2} \geq i n f_{i \in I} K_{i} \sum_{i \in I}\left\|G_{i} f\right\|^{2} \\
\geq i n f_{i \in I} K_{i} A\|f\|^{2} .
\end{gathered}
$$

Therefore, the sequence $\left\{D_{i} G_{i}\right\}$ is a $G$-frame.

Definition 3.2. A $G$-frame $\left\{G_{i} \in \mathcal{L}\left(\mathcal{H}, \mathcal{H}_{i}\right)\right\}_{i \in I}$ is called scalable $G$-frame for $\mathcal{H}$ with respect to $\left\{\mathcal{H}_{i}\right\}$ whenever there exists a sequence of non-negative diagonal operators $\left\{D_{i} \in L\left(\mathcal{H}_{i}\right)\right\}_{i \in I}$ such that $\left\{D_{i} G_{i}\right\}$ is a Parseval $G$-frame. Also, if $D_{i}$ 's are positive operators then the $G$-frame $\left\{G_{i}\right\}_{i \in I}$ is called strictly scalable $G$-frame.

Here are some examples of the scalability of $G$-frames for better understanding of the definition. The following example shows that the new definition of scalability is a generalization of the definition of scalability.

Example 3.1. Let $\left\{f_{i}\right\}_{i \in \mathbb{I}}$ be a scalable frame for $\mathcal{H}$ with the sequence of positive scalars $\left\{\alpha_{i}\right\}_{i \in \mathbb{I}}$. For each $i \in I$, set $D_{i}=\left[\alpha_{i}\right]$, where $\left[\alpha_{i}\right]$ is an one-by-one matrix. Then the $G$-frame $\left\{G_{i}\right\}$ where $G_{i}: \mathcal{H} \rightarrow \mathbb{C}$ defined by $G_{i} f=\left\langle f, f_{i}\right\rangle$ for each $f \in \mathcal{H}$, is a scalable $G$-frame for $\mathcal{H}$ with respect to $\mathbb{C}$ with diagonal operators $\left\{D_{i}\right\}$, because

$$
\sum_{i \in I}\left\|D_{i} G_{i} f\right\|^{2}=\sum_{i \in I}\left|\alpha_{i}\left\langle f, f_{i}\right\rangle\right|^{2}=\|f\|^{2} \quad \forall f \in \mathcal{H} .
$$

Example 3.2. Let $\mathcal{H}$ be a separable Hilbert space and $\left\{\mathcal{H}_{i}\right\}$ be a sequence of separable Hilbert subspaces such that $\mathcal{H}=\bigoplus_{i} \mathcal{H}_{i}$. Also, let $\left\{f_{i j}\right\}_{j \in \mathbb{K}_{i}}$ be a scalable frame for $\mathcal{H}_{i}$. Then $\left\{G_{i} \in \mathcal{L}\left(\mathcal{H}, \mathcal{H}_{i}\right)\right\}_{i \in I}$ such that $G_{i} f=\sum_{j \in \mathbb{K}_{i}}\left\langle f, f_{i, j}\right\rangle f_{i, j}$ for each $f \in \mathcal{H}$, is a scalable $G$ - frame.

Example 3.3. Let $\mathcal{H}=\mathbb{C}^{3}$, and $\mathcal{H}_{1}=\mathcal{H}_{2}=\mathcal{H}_{3}=\mathbb{C}^{2}$. Define

$$
G_{1}=\left[\begin{array}{ccc}
-1 & 0 & 0 \\
1 & 0 & 0
\end{array}\right] \quad G_{2}=\left[\begin{array}{ccc}
0 & 1 & 0 \\
0 & -1 & 0
\end{array}\right] \quad G_{3}=\left[\begin{array}{lll}
0 & 0 & 1 \\
0 & 0 & 1
\end{array}\right]
$$

and

$$
D_{1}=\left[\begin{array}{ll}
0 & 0 \\
0 & 1
\end{array}\right] \quad D_{2}=\left[\begin{array}{ll}
1 & 0 \\
0 & 0
\end{array}\right] \quad D_{3}=\left[\begin{array}{cc}
\frac{1}{\sqrt{2}} & 0 \\
0 & \frac{1}{\sqrt{2}}
\end{array}\right]
$$


Now, we have

$$
\sum_{i=1}^{3}\left\|D_{i} G_{i} f\right\|^{2}=\left|z_{1}\right|^{2}+\left|z_{2}\right|^{2}+\left|z_{3}\right|^{2}=\|f\|^{2}, \quad \forall f=\left(z_{1}, z_{2}, z_{3}\right) \in \mathbb{C}^{3} .
$$

To answer the question that was first raised in the section, we present a theorem that states the necessary and sufficient conditions for $G$-framing with the help of a block diagonal operator.

Theorem 3.1. Let $\mathcal{G}=\left\{G_{i} \in \mathcal{L}\left(\mathcal{H}, \mathcal{H}_{i}\right)\right\}_{i \in I}$ be a $G$-frame for the Hilbert space $\mathcal{H}$ with respect to $\left\{\mathcal{H}_{i}\right\}_{i \in I}$ with analysis operator $T_{\mathcal{G}}^{*}$. Also, assume that $\left\{D_{i} \in\right.$ $\left.L\left(\mathcal{H}_{i}\right)\right\}_{i \in I}$ is a sequence of non-negative diagonal operators. Then $H=\left\{D_{i} G_{i}\right\}_{i \in I}$ is a $G$-frame if and only if rang $T_{\mathcal{G}}^{*} \subseteq \operatorname{dom} D,\left.D\right|_{\text {rang }} T_{\mathcal{G}}^{*}$ is $I C R$, where $D$ is a bounded block diagonal operator on $\left(\bigoplus \mathcal{H}_{i}\right)_{l^{2}}$ such that $\left\{D_{i}\right\}_{i \in I}$ is as its diagonal.

Proof. Let $H=\left\{D_{i} G_{i}\right\}_{i \in I}$ be a $G$-frame and $T_{\mathcal{G}}^{*}$ be analysis operator of $\mathcal{G}=$ $\left\{G_{i}\right\}_{i \in I}$.

$$
\left(T_{\mathcal{G}}^{*} f\right)_{j}=D_{j} G_{j} f=\left(D G_{j} f\right)_{j}
$$

Thus, $T_{H}^{*}=D T_{\mathcal{G}}^{*}$. Since $\operatorname{dom} T_{H}^{*}=\mathcal{H}$ then $\operatorname{rang} T_{\mathcal{G}}^{*} \subseteq \operatorname{dom} D$. Also, since $\mathcal{G}$ is a $G$-frame, then $\operatorname{rang}_{\mathcal{G}}^{*}$ is closed. On the other hand, due to $H$ is a $G$-frame, there exist $A_{1}, B_{1}>0$ such that for each $f \in \mathcal{H}$

$$
A_{1}\|f\|^{2} \leq\left\|T_{H}^{*} f\right\|_{l^{2}}^{2} \leq B_{1}\|f\|^{2} .
$$

Set $\nu=T_{\mathcal{G}}^{*} f \in \operatorname{rang} T_{\mathcal{G}}^{*}$ so,

$$
\|D \nu\|^{2}=\left\|D T_{\mathcal{G}}^{*} f\right\|^{2} \geq A_{1}\|f\|^{2} \geq A_{1}\left\|T_{\mathcal{G}}^{*}\right\|^{-2}\|\nu\|^{2} .
$$

Thus $\left.D\right|_{\text {rang } T_{\mathcal{G}}^{*}}$ is ICR.

Conversely, let $\left.D\right|_{\text {rang }} T_{\mathcal{G}}^{*}$ be ICR and $\operatorname{rang} T_{\mathcal{G}}^{*} \subset \operatorname{dom} D$. Due to the closed graph theorem, the operator $\left.D\right|_{r} a n g T_{\mathcal{G}}^{*}$ is a bounded operator and since $\left.D\right|_{\text {rang } T_{\mathcal{G}}^{*}}$ is ICR, there exist two constants $a, b>0$ such that

$$
a\|y\|^{2} \leq\|D y\|^{2} \leq b\|y\|^{2}, \quad \forall y \in \operatorname{rang} T_{\mathcal{G}}^{*} .
$$

On the other hand, we know that $T_{\mathcal{G}}^{*}$ is bounded and ICR, thus there exist two constants $a^{\prime}, b^{\prime}>0$ such that

$$
a^{\prime}\|f\|^{2} \leq\left\|T_{\mathcal{G}}^{*} f\right\|^{2} \leq b^{\prime}\|f\|^{2}, \quad \forall f \in \mathcal{H} .
$$

By using (3.1) and ( 3.2) we have

$$
a a^{\prime}\|f\|^{2} \leq\left\|D T_{\mathcal{G}}^{*} f\right\|^{2} \leq b b^{\prime}\|f\|^{2}, \quad \forall f \in \mathcal{H} .
$$

Therefore $H$ is a $G$-frame.

Also, due to the boundedness of $D T_{\mathcal{G}}^{*}$, it is proved that $\left(D T_{\mathcal{G}}^{*}\right)^{*}=\overline{T_{\mathcal{G}} D}$, which completes the proof.

The block diagonal operator is not necessarily bounded. In the next proposition, we state the necessary and sufficient conditions for this operator to be bounded.

Proposition 3.1. Let $\mathcal{G}=\left\{G_{i} \in \mathcal{L}\left(\mathcal{H}, \mathcal{H}_{i}\right)\right\}_{i \in I}$ be a $G$-frame for the Hilbert space $\mathcal{H}$ with respect to $\left\{\mathcal{H}_{i}\right\}_{i \in I}$ such that $G_{i}$ 's be bounded below and liminf $\left\|G_{i}\right\|>0$. Also, assume that on $\left\{D_{i} \in L\left(\mathcal{H}_{i}\right)\right\}_{i \in I}$ is a sequence of non-negative diagonal operators. Then $\mathcal{F}=\left\{D_{i} G_{i}\right\}_{i \in I}$ is a $G$-frame if and only if the block diagonal 
operator $D$ with $D_{i}$ as blocks is bounded and $\left.D\right|_{\text {rangT }} T_{\mathcal{G}}^{*}$ is ICR. In this case $S_{\mathcal{F}}=$ $T_{\mathcal{G}} D^{2} T_{\mathcal{G}}^{*}$.

Proof. Let $\mathcal{F}=\left\{D_{i} G_{i}\right\}_{i \in I}$ be a $G$-frame. Then $\mathcal{F}=\left\{D_{i} G_{i}\right\}_{i \in I}$ is $G$-Bessel. So, there exists a positive constant $B$ such that for each $f \in \mathcal{H}$,

$$
\sum_{i \in I}\left\|D_{i} G_{i} f\right\|^{2} \leq B\|f\|^{2} .
$$

Now,

$$
\left\|G_{i} f\right\|^{2} \leq\left\|D_{i}\right\|^{-2}\left\|D_{i} G_{i} f\right\|^{2} \leq\left\|D_{i}\right\|^{-2} B\|f\|^{2} .
$$

Since $G_{i}$ 's are bounded below so, for each $i \in I$, there exists a $A_{i}>0$ such that $A_{i}\|f\|^{2} \leq\left\|G_{i} f\right\|^{2}$ for each $f \in \mathcal{H}$. Also, due to $\liminf \left\|G_{i}\right\|>0$ there exists a $\delta>0$ and $j \in I$ such that $A_{i} \geq \delta$ for each $i \geq j$. So, by $(3.4),\left\|D_{i}\right\| \leq(B \delta)^{1 / 2}$ for each $i \geq j$. Therefore, there exists an $M>0$ such that for each $i,\left\|D_{i}\right\| \leq M$. That is $\|D\|<\infty$. By Theorem 3.1, the converse is clear.

Using the results of Proposition 3.1 and Theorem 3.1, the following proposition is obtained.

Proposition 3.2. Let $\mathcal{G}=\left\{G_{i}\right\}_{i \in I}$ be a $G$-frame with the assumptions of Proposition 3.1 and analysis operator $T_{\mathcal{G}}^{*}$. Then $\mathcal{G}$ is scalable $G$-frame if and only if there exists a bounded block diagonal operator $D$ on $\left(\bigoplus \mathcal{H}_{i}\right)_{l^{2}}$ such that

$$
T_{\mathcal{G}} D^{2} T_{\mathcal{G}}^{*}=I_{\mathcal{H}}
$$

Proof. Let $\mathcal{G}=\left\{G_{i}\right\}_{i \in I}$ be a scalable $G$-frame, so there exists a sequence $\left\{D_{i} \in\right.$ $\left.L\left(\mathcal{H}_{i}\right)\right\}_{i \in I}$ of non-negative diagonal operators such that $\mathcal{F}=\left\{D_{i} G_{i}\right\}_{i \in I}$ is a Parseval $G$-frame.

We define $D$ as a block diagonal operator on $\left(\bigoplus \mathcal{H}_{i}\right)_{l^{2}}$ which $\left\{D_{i}\right\}_{i \in I}$ is as its diagonal blocks. By Theorem 3.1 we have

$$
\operatorname{rang} T_{\mathcal{G}}^{*} \subseteq \operatorname{dom} D, S_{\mathcal{F}}=T_{\mathcal{G}} D^{2} T_{\mathcal{G}}^{*}
$$

Since $\mathcal{F}$ is a Parseval frame then $S_{\mathcal{F}}=I_{\mathcal{H}}$.

Conversely, assume that there exists a block diagonal operator $D$ on $\left(\bigoplus \mathcal{H}_{i}\right)_{l^{2}}$ with blocks $\left\{D_{i} \in L\left(\mathcal{H}_{i}\right)\right\}_{i \in I}$ such that $T_{\mathcal{G}} D^{2} T_{\mathcal{G}}^{*}=I_{\mathcal{H}}$. This concludes that rang $T_{\mathcal{G}}^{*} \subseteq \operatorname{dom} D$. Also, $D T_{\mathcal{G}}^{*}$ is closed operator from $\mathcal{H}$ into $\left(\bigoplus \mathcal{H}_{i}\right)_{l^{2}}$ due to $D$ and $T_{\mathcal{G}}^{*}$ are bounded operators. This implies that $\left.D\right|_{\text {rang } T_{\mathcal{G}}^{*}}$ is ICR.

Besides, since $T_{\mathcal{G}} D^{2} T_{\mathcal{G}}^{*}=I_{\mathcal{H}}$ then $D T_{\mathcal{G}}^{*}$ is isometric. Now using by Proposition 3.1 , we conclude that $\mathcal{F}$ is a Parseval $G$-frame.

As we have seen [17], a frame induced by a $G$-frame inherits the desired properties of the $G$-frame. In the following, we will describe another of these desirable properties that inherits the frame induced by $G$-frame.

Theorem 3.2. Let $\left\{G_{i} \in \mathcal{L}\left(\mathcal{H}, \mathcal{H}_{i}\right)\right\}_{i \in I}$ be a $G$-frame for the Hilbert space $\mathcal{H}$ with respect to $\left\{\mathcal{H}_{i}\right\}_{i \in I}$ and $\left\{u_{i, j}\right\}_{i \in I, j \in \mathbb{K}_{i}}$ be the frame induced by the $G$-frame $\left\{G_{i}\right\}_{i \in I}$, then $\left\{G_{i}\right\}_{i \in I}$ is scalable $G$-frame if and only if $\left\{u_{i, j}\right\}_{i \in I, j \in \mathbb{K}_{i}}$ is scalable frame.

Proof. Let $\left\{G_{i}\right\}_{i \in I}$ be a scalable $G$-frame. Then there exists a sequence of nonnegative diagonal operators $\left\{D_{i}\right\}_{i \in I}$ such that $\left\{D_{i} G_{i}\right\}_{i \in I}$ is a Parseval frame. By definition of diagonal operators there exists $\left\{\alpha_{i, j}\right\}_{i \in I, j \in \mathbb{K}_{i}}$ of non-negative scalars 
such that $D_{i} e_{i, j}=\alpha_{i, j} e_{i, j}$ for all $i \in I$ and $j \in \mathbb{K}_{i}$, where, for each $i \in I$, the sequence $\left\{e_{i, j}\right\}_{j \in \mathbb{K}_{i}}$ is an orthonormal basis for $\mathcal{H}_{i}$.

Thus

$$
\|f\|^{2}=\sum_{i \in I}\left\|D_{i} G_{i} f\right\|^{2}=\sum_{i \in I} \sum_{j \in \mathbb{K}_{i}}\left|\left\langle f, \alpha_{i, j} u_{i, j}\right\rangle\right|^{2} .
$$

That is the desired result.

For the converse, let $\left\{u_{i, j}\right\}_{i \in I, j \in \mathbb{K}_{i}}$ be a scalable frame for the Hilbert space $\mathcal{H}$, then there exists a sequence of non-negative scalars $\left\{\alpha_{i, j}\right\}_{i \in I, j \in \mathbb{K}_{i}}$ such that $\left\{\alpha_{i, j} u_{i, j}\right\}_{i \in I, j \in \mathbb{K}_{i}}$ is a Parseval frame for Hilbert space $\mathcal{H}$. Set $D_{i} e_{i, j}=\alpha_{i, j} e_{i, j} ; i \in$ $I, \quad j \in \mathbb{K}_{i}$, thus

$$
\|f\|^{2}=\sum_{i \in I}\left\|D_{i} G_{i} f\right\|^{2}=\sum_{i \in I} \sum_{j \in \mathbb{K}_{i}}\left|\left\langle f, \alpha_{i, j} u_{i, j}\right\rangle\right|^{2} .
$$

In analogy to Theorem 2.1, we prove an equivalent condition to show that a $G$-frame is not scalable $G$-frame.

Proposition 3.3. Let $\mathcal{G}=\left\{G_{i} \in \mathcal{L}\left(\mathbb{R}^{N}, \mathbb{R}\right)\right\}_{i=1}^{M}$ be a $G$-frame for the Hilbert space $\mathbb{R}^{N}$ with respect to $\mathbb{R}$. Then $\mathcal{G}$ is not a scalable $G$-frame if and only if there exists a symmetric matrix $Y$ on $\mathbb{R}^{N \times N}$ such that $\operatorname{tr}(Y)<0$ and $\left[G_{i}^{*} e_{i j}\right]^{T} Y\left[G_{i}^{*} e_{i j}\right] \geq 0$, where $\left[G_{i}^{*} e_{i j}\right]^{T}$ denotes the transpose of the matrix $\left[G_{i}^{*} e_{i j}\right]$.

Proof. $\left\{G_{i}\right\}_{i=1}^{M}$ is not a scalable $G$-frame if and only if the frame $\left\{u_{i j}\right\}$ is not scalable frame for $\mathbb{R}^{N}$. By Theorem 2.1 there exists a symmetric matrix $Y$ on $\mathbb{R}^{N \times N}$ such that $\operatorname{tr}(Y)<0$ and $\left[u_{i j}\right]^{T} Y\left[u_{i j}\right] \geq 0$. This means there exists a symmetric matrix $Y$ on $\mathbb{R}^{N \times N}$ such that $\operatorname{tr}(Y)<0$ and $\left[G_{i}^{*} e_{i j}\right]^{T} Y\left[G_{i}^{*} e_{i j}\right] \geq 0$.

\section{New structures of scalable $G$-frames}

In this section, we will describe new structures for scalable $G$-frames.

Proposition 4.1. Let $\left\{G_{i} \in \mathcal{L}\left(\mathcal{H}, \mathcal{H}_{i}\right)\right\}_{i \in I}$ be a scalable $G$-frame for the Hilbert space $\mathcal{H}$ with respect to $\left\{\mathcal{H}_{i}\right\}_{i \in I}$ and $U: \mathcal{H} \rightarrow \mathcal{H}$ be a bounded linear operator then $\left\{G_{i} U\right\}_{i \in I}$ is scalable $G$-frame if and only if $U: \mathcal{H} \rightarrow \mathcal{H}$ is a unitary operator.

Proof. Since $\left\{G_{i}\right\}_{i \in I}$ is a scalable $G$-frame, then there exists a sequence $\left\{D_{i} \in\right.$ $\left.L\left(\mathcal{H}_{i}\right)\right\}_{i \in I}$ of non-negative diagonal operators such that $\left\{D_{i} G_{i}\right\}_{i \in I}$ is Parseval $G$-frame.

Therefore,

$$
\sum_{i \in I}\left\|D_{i} G_{i} U f\right\|^{2}=\|U f\|^{2} .
$$

Thus, $\left\{G_{i} U\right\}_{i \in I}$ is scalable $G$-frame if and only if $\|U f\|=\|f\|$. This completes the proof.

Let $\mathcal{H}$ and $\mathcal{K}$ be two Hilbert spaces. The direct sum of Hilbert spaces $\mathcal{H}$ and $\mathcal{K}$ is denoted by $\mathcal{H} \oplus \mathcal{K}=\{(h, k): h \in \mathcal{H}, k \in \mathcal{K}\}$, which is a Hilbert space with pointwise operations and inner product

$$
\left\langle(f, g),\left(f^{\prime}, g^{\prime}\right)\right\rangle=\left\langle f, f^{\prime}\right\rangle_{\mathcal{H}}+\left\langle g, g^{\prime}\right\rangle_{\mathcal{K}}, \quad \forall f, f^{\prime} \in \mathcal{H}, \quad \forall g, g^{\prime} \in \mathcal{K} .
$$


If $U$ and $W$ are Hilbert spaces and $G \in \mathcal{L}(\mathcal{H}, U), T \in \mathcal{L}(\mathcal{K}, W)$, we recall that

$$
G \oplus T \in \mathcal{L}(\mathcal{H} \oplus \mathcal{K}, U \oplus W) \quad \text { by } G \oplus T(h, k)=(G h, T k) \quad \forall h \in \mathcal{H}, \quad k \in \mathcal{K} .
$$

Refer to $[9,12]$ for more information.

Theorem 4.1. Let $\left\{G_{i} \in \mathcal{L}\left(\mathcal{H}, \mathcal{H}_{i}\right)\right\}_{i \in I}$ be a scalable $G$-frame for $\mathcal{H}$ with respect to $\left\{\mathcal{H}_{i}\right\}_{i \in I}$ and $\left\{G_{i}^{\prime} \in \mathcal{L}\left(\mathcal{K}, \mathcal{K}_{i}\right)\right\}_{i \in I}$ be a scalable $G$-frame for $\mathcal{K}$ with respect to $\left\{\mathcal{K}_{i}\right\}_{i \in I}$. Then $\left\{G_{i} \oplus G_{i}^{\prime} \in \mathcal{L}\left(\mathcal{H} \oplus \mathcal{K}, \mathcal{H}_{i} \oplus \mathcal{K}_{i}\right)\right\}_{i \in I}$ is scalable $G$-frame.

Proof. Since $\left\{G_{i}\right\}_{i \in I}$ is a scalable $G$-frame, there exists a sequence $\left\{D_{i} \in L\left(\mathcal{H}_{i}\right)\right\}_{i \in I}$ of non-negative diagonal operators such that $\left\{D_{i} G_{i}\right\}_{i \in I}$ is a Parseval $G$-frame. Also, there exists a sequence $\left\{D_{i}^{\prime} \in L\left(\mathcal{K}_{i}\right)\right\}_{i \in I}$ of non-negative diagonal operators such that $\left\{D_{i}^{\prime} G_{i}^{\prime}\right\}_{i \in I}$ is a Parseval $G$-frame.

Now, set $S_{i}=\left[\begin{array}{cc}D_{i} & 0 \\ 0 & D_{i}^{\prime}\end{array}\right], i \in I$. The sequence $\left\{S_{i} \in L\left(\mathcal{H}_{i} \oplus \mathcal{K}_{i}\right)\right\}_{i \in I}$ is a sequence of non-negative diagonal operators on $\mathcal{H}_{i} \oplus \mathcal{K}_{i}$.

Therefore

$$
\begin{gathered}
\sum_{i \in I}\left\|S_{i} G_{i} \oplus G_{i}^{\prime}(f, g)\right\|^{2}=\sum_{i \in I}\left\|D_{i} G_{i}(f)\right\|^{2}+\sum_{i \in I}\left\|D_{i}^{\prime} G_{i}^{\prime}(g)\right\|^{2} \\
=\|f\|^{2}+\|g\|^{2}=\|(f, g)\|^{2} \quad \forall(f, g) \in \mathcal{H} \oplus \mathcal{K} .
\end{gathered}
$$

Corollary 4.1. Let $\left\{G_{i} \in \mathcal{L}\left(\mathcal{H}, \mathcal{H}_{i}\right)\right\}_{i \in I}$ be scalable $G$-frame for $\mathcal{H}$ with respect to $\left\{\mathcal{H}_{i}\right\}_{i \in I}$ and $\left\{G_{i}^{\prime} \in \mathcal{L}\left(\mathcal{K}, \mathcal{K}_{i}\right)\right\}_{i \in I}$ be A-tight $G$-frame for $\mathcal{K}$ with respect to $\left\{\mathcal{K}_{i}\right\}_{i \in I}$. Then $\left\{G_{i} \oplus G_{i}^{\prime} \in \mathcal{L}\left(\mathcal{H} \oplus \mathcal{K}, \mathcal{H}_{i} \oplus \mathcal{K}_{i}\right)\right\}_{i \in I}$ is a scalable $G$-frame.

Theorem 4.2. Let $\left\{G_{i} \in \mathcal{L}\left(\mathcal{H}, \mathcal{H}_{i}\right)\right\}_{i \in I}$ be a $G$-frame for $\mathcal{H}$ with respect to $\left\{\mathcal{H}_{i}\right\}_{i \in I}$ and for each $i \in I,\left\{T_{i, j} \in \mathcal{L}\left(\mathcal{H}_{i}, \mathcal{K}_{i, j}\right)\right\}_{j \in J}$ be a scalable $G$-frame for $\mathcal{H}_{i}$ with respect to $\left\{\mathcal{K}_{i, j}\right\}_{j \in J}$ by a sequence of non-negative diagonal operators $\left\{D_{i, j} \in L\left(\mathcal{K}_{i, j}\right)\right\}_{j \in J}$. Then $\left\{T_{i, j} G_{i}\right\}_{i \in I, j \in J}$ is a scalable $G$-frame for $\mathcal{H}$ with respect to $\left\{\mathcal{K}_{i, j}\right\}_{i \in I, j \in J}$ by the sequence $\left\{D_{i, j} \in L\left(\mathcal{K}_{i, j}\right)\right\}_{i \in I, j \in J}$ if and only if $\left\{G_{i}\right\}_{i \in I}$ is a Parseval $G$-frame.

Proof. Let $i \in I$. Under the above assumptions, $\left\{T_{i, j}\right\}_{j \in J}$ is a scalable $G$-frame for $\mathcal{H}_{i}$ with respect to $\left\{\mathcal{K}_{i, j}\right\}_{j \in J}$ by a sequence of non-negative diagonal operators $\left\{D_{i, j} \in L\left(\mathcal{K}_{i, j}\right)\right\}_{j \in J}$. Then

$$
\|f\|^{2}=\sum_{j \in J}\left\|D_{i, j} T_{i, j} f\right\|^{2} \quad \forall f \in \mathcal{K}_{i, j} .
$$

Now, assume that $\left\{T_{i j} G_{i}\right\}_{i \in I, j \in J}$ is a scalable $G$-frame for $\mathcal{H}$ with respect to $\left\{\mathcal{K}_{i j}\right\}_{i \in I, j \in J}$ and the sequence $\left\{D_{i, j}\right\}_{i \in I, j \in J}$ of non-negative diagonal operators, for which the $G$-frame $\left\{D_{i, j} T_{i, j} G_{i}\right\}_{i \in I, j \in J}$ is Parseval $G$-frame. Hence, by (4.1)

$$
\|f\|^{2}=\sum_{i \in I, j \in J}\left\|D_{i, j} T_{i, j} G_{i} f\right\|^{2}=\sum_{i \in I}\left\|G_{i} f\right\|^{2} \quad \forall f \in \mathcal{H} .
$$

Therefore $\left\{G_{i}\right\}_{i \in I}$ is Parseval $G$-frame. 
Conversely, assume that $\left\{G_{i}\right\}_{i \in I}$ is Parseval $G$-frame. For each $i \in I,\left\{T_{i, j} G_{i}\right\}_{j \in J}$ is a scalable $G$-frame by a sequence of non-negative diagonal operators $\left\{D_{i, j} \in\right.$ $\left.L\left(\mathcal{K}_{i, j}\right)\right\}_{j \in J}$ for $\mathcal{H}_{i}$ with respect to $\left\{\mathcal{K}_{i, j}\right\}_{j \in J}$; thus for all $f \in \mathcal{H}$,

$$
\sum_{i \in I, j \in J}\left\|D_{i, j} T_{i, j} G_{i} f\right\|^{2}=\sum_{i \in I}\left\|G_{i} f\right\|^{2}=\|f\|^{2} .
$$

This completes the proof.

Using the previous theorem, we can say that if any Hilbert space $\mathcal{H}_{i}$ has a scalable frame, then there exists a scalable $G$-frame for $\mathcal{H}$ with respect to $\left\{\mathcal{H}_{i}\right\}_{i \in I}$.

\section{Acknowledgements}

The authors would like to thank the reviewers for careful reading of the manuscript and giving useful comments, which helped to improve the paper.

\section{References}

[1] P. Casazza and X. Chen, Frame scalings: A condition number approach, Linear Algebra and Appl. $\mathbf{5 2 3}$ (2017), 152-168.

[2] P. Casazza and G. Kutyniok, Finite Frames: Theory and Applications, Brikhauser, Boston, 2013.

[3] X. Chen, G. Kutyniok, K. A. Okoudjou, F. Philipp, and R. Wang, Measures of scalability, IEEE Trans. Inf. Theory, 8 (2015), 4410-4423.

[4] O. Christensen, Frames and Bases, Brikhauser, Boston, 2008.

[5] O. Christensen and Y. C. Eldar, Oblique dual frames and shift invariant spaces, Appl. Comput. Harmonic Anal. 17(2004), 48-68.

[6] J. Chun Hou, A characterization of positive elementary operators, J. Operator Theory 1 (1998), 43-58.

[7] I. Daubechies, A. Grossmann, and Y. Meyer, Painless nonorthogonal expansions, $J$. Math. Phys. 27 (1986), 1271-1283.

[8] R.J. Duffin and A.C. Schaeffer, A class of nonharmonic Fourier series, Trans. Amer. Math. Soc. 72 (1952), 341-366.

[9] G. B. Folland, A Course in Abstract Harmonic Analysis, CRC Press BOCA Raton, Florida, 1995.

[10] M. Fornasier, Quasi-Orthogonal decompositions of structured frames, J. Math. Anal. Appl. 289 (2004), 180-199.

[11] D. Gabor, Theory of communication. Part 1, The analysis of information, J. IEE, London 93 (1946), 429-457.

[12] A. Khosravi, K. Khosravi, Fusion frames and G-frames, J. Math. Anal. Appl. 342 (2008), 1068-1083.

[13] G. Kutyniok , K. Okoudjou, F. Philipp, and E.K. Tuley, Scalable frames, Linear Algebra and Appl. 5 (2013), 2225-2238.

[14] G. Kutyniok, K. Okoudjou, and F. Philipp, Scalable frames and convex geometry, Contemp. Math. 626 (2014), 19-32.

[15] S. Li and H. Ogawa, Pseudo frames for subspaces with applications, J.Fourier Anal. Appl. 10 (2004), 409-431.

[16] A. Okoudjou. Preconditioning techniques in frame theory and probabilistic frames, Proceedings of Symposia in Applied Mathematics 73 (2016), 105-142.

[17] W. Sun, G-frames and g-Riesz bases, J. Math. Anal. Appl. 322(2006), 437-452.

[18] S. F. D. Waldron, An Introduction to Finite Tight Frames, Birkhauser, Boston, 2017. 
Ahmad Ahmadi

Department of Mathematics, University of Hormozgan, Bandar Abbas, Iran. E-mail address: ahmadi_a@hormozgan.ac.ir

\author{
Asghar Rahimi \\ Department of Mathematics, University of Maragheh, Maragheh, Iran. \\ E-mail address: rahimi@maragheh.ac.ir
}

Received: May 22, 2020; Revised: February 24, 2021; Accepted: April 5, 2021 